\title{
Inhibition of Return after Color Singletons
}

\author{
Heinz-Werner Priess \\ University of Vienna \\ Sabine Born \\ Université de Genève \\ Ulrich Ansorge
University of Vienna
}

\begin{abstract}
Inhibition of return (IOR) is the faster selection of hitherto unattended than previously attended positions. Some previous studies failed to find evidence for IOR after attention capture by color singletons. Others, however, did report IOR effects after color singletons. The current study examines the role of cue relevance for obtaining IOR effects. By using a potentially more sensitive method - saccadic IOR - we tested and found IOR after relevant color singleton cues that required an attention shift (Experiment 1). In contrast, irrelevant color singletons failed to produce reliable IOR effects in Experiment 2. Also, Experiment 2 rules out an alternative explanation of our IOR findings in terms of masking. We discuss our results in light of pertaining theories of IOR.
\end{abstract}

\section{Keywords: Inhibition of return, attention, singleton capture, saccades, eye movements}

\section{Introduction}

When we look around, only a part of the visual signals arriving at our retinae at each moment in time is selected for in-depth processing. This form of selectivity is called visual attention. Visual attention has many different purposes. Selection can gate access of information to perception (Scharlau, 2002), memory (Duncan \& Humphreys, 1989), or motor control (Allport, 1989). Corresponding to its multiple purposes, visual attention is also influenced by many different factors, such as the properties of the stimulus (Itti, Koch, \& Niebur, 1998) and the goals of the human observer (Folk, Remington, \& Johnston, 1992). One important factor is the observer's memory trace of the visual information that has been selected in the recent past. One primitive form of such memory has been called inhibition of return (IOR): It has been demonstrated that shifting visual attention toward one position in space delays a second subsequent attention shift to the same position at a later point in time (Posner \& Cohen, 1984; Taylor \& Klein, 1998). This phenomenon is called IOR because it is supposed to reflect the human resistance to select information from po- sitions that have recently already been inspected by a covert shift of attention (Posner \& Cohen, 1984). In this context, a covert shift of attention is a shift of attention without the eyes, whereas an overt shift would be accompanied by an eye movement (Posner, 1980).

Understanding the principles governing IOR is mandatory for a broader understanding of attention. The conclusions that are drawn from IOR research are not always the same as the conclusions which are reached in research on single attention shifts (e.g., Pratt, Sekuler, \& McAuliffe, 2001). Accordingly, some neurophysiological studies suggest that attention capture and IOR arise at different stages of processing (e.g., Fecteau \& Munoz, 2005). IOR research therefore complements the picture of the operation of attention.

Since IOR's original discovery, many factors were identified that influence the magnitude of IOR. Some authors believe that IOR mostly reflects selection-foraction because it is particularly strong with overt shifts that is, with saccades, the fast jumping movements of the eyes, whereas IOR is weaker with a covert attention shift (e.g., starts later, Pratt \& Neggers, 2008) and can even be absent with some forms of covert attention (Klein \& Pon- 
tefract, 1994; Rafal, Calabresi, Brennan, \& Sciolto, 1989; see Taylor \& Klein, 2000 for a review).

The current study was motivated by observations from Pratt et al. (2001) and others of massive differences in the magnitude of IOR after attention shifts to abrupt onset stimuli versus color singletons. Both, abrupt onsets in the periphery and singletons popping out among a background of more homogenous color stimuli (such as a green apple among red apples) have a strong potential to capture human attention. Some researchers believe that this capture is truly automatic and stimulus-driven (see Theeuwes, 2010, for a review). Others are more reserved and regard it as being conditional on top-down search goals (Folk et al., 1992).

Whichever of these two positions holds true: important in the present context is that some authors claimed to have found IOR after color singletons (Godijn \& Theeuwes, 2004; Theeuwes \& Chen, 2005; Theeuwes \& Godijn, 2002), whereas others did not find IOR after color singletons (Gibson \& Amelio, 2000; Pratt et al., 20001). All of these authors used cueing paradigms with peripheral cues presented either at the same position (SP -also called the "valid condition" by some authors; cf. Posner, 1980) as the subsequent target or at a different position (DP -also called the "invalid condition"; cf. Posner, 1980) than the target. For example, Pratt et al. (2001) used two kinds of uninformative cues, onset cues and color-singleton cues. The onset cue was a single onset stimulus (four white dots abruptly appearing around one of four placeholder squares). The color-singleton cue consisted of four red dots appearing around one of the four placeholders, whereas white dots appeared around the remaining three placeholders. Two cue-target intervals were used: one short $(150 \mathrm{~ms})$ and one long $(850$ $\mathrm{ms})$. With the long cue-target interval the authors only found IOR after onset cues. Reaction Times (RTs) were slower for SP than for DP targets. With the colorsingleton cues, however, no IOR was found (see also Gibson \& Amelio, 2000). These results seem to support the view that onsets are special in that they created stronger selection-for-action effects, maybe because onset-driven attention capture depends on color-insensitive processing in the midbrain's superior colliculi (SC) (Fuchs \& Ansorge, 2012). The SC are strongly involved in the programming and in the execution of eye movements (Wurtz \& Albano, 1980) and many results imply that onsets might have a higher ability than for example color to activate the eye-movement system (Wu \& Remington, 2003). In sum, some studies suggest that IOR could be restricted to attention capture by abrupt onsets and may not be observed with color singletons.

In contrast, other authors have reported IOR effects after color singletons (Godijn \& Theeuwes, 2004; Theeuwes \& Chen, 2005; Theeuwes \& Godijn, 2002). In these studies the procedures or measures were slightly different from the classic cuing paradigm that most IOR studies use. For example, Theeuwes and Godijn (2002) used a manual go-no-go target detection task and found that a color-singleton cue produced IOR in RTs of gotrials. The go-no-go task is controversial, however, because it is uncertain whether the singleton-cueing effect (and accordingly the IOR effect) in the go-no-go task reflects attention. This doubt stems from a lack of an $\mathrm{N} 2 \mathrm{pc}$, the typical event-related potentials (ERPs) indicative of an attention shift. Whereas attention-capture by color singletons usually elicits an N2pc (Eimer \& Kiss, 2008; Hickey, MacDonald, Theeuwes, \& 2006), the N2pc effect can be missing with go-no-go decisions (Adamo, Pun, \& Ferber, 2010). In another study, Theeuwes and Chen (2005) used $d$ ' as a measure of attention allocation. This is a classic measure of target discrimination. Thus, at face value, singleton-cueing and IOR effects on $d$, would be uncontroversial reflections of attention. However, because Theeuwes and Chen did not report RTs in SP and DP conditions, it is possible that, for instance, a tendency to trade off discrimination accuracy for discrimination speed was reflected in their color-singleton cueing and IOR effects. Finally, Godijn and Theeuwes (2004) used a paradigm in which a first vertical saccade (either up or down) had to be made according to a color singleton cue. When analyzing subsequent saccades (i.e., saccades made according to a second cue after participants had returned to central fixation) either towards or away from that initial cue, they found a small IOR effect in saccadic RTs.

Godijn and Theeuwes (2004) argue that the discrepancy of their results with other studies finding no IOR after color singletons might have been due to two factors: First most other research has tested whether color singletons produce IOR with manual responses (Ansorge \& Heumann, 2004; Gibson \& Amelio, 2000; Pratt et al., 2001; Remington, Folk, \& McLean, 2001). Second, the cues in their study were task-relevant and could not be 
ignored. In contrast, most other studies used uninformative cues.

In the present study we therefore set out to test whether cue relevance is important to obtain saccadic IOR after color singletons. In fact, evidence for IOR being modulated by task-relevance of a cue comes from Gibson and Amelio (2000). They used targets that were characterized by abrupt onsets or targets that were color singletons. Importantly, these authors have found that onset cues only captured attention and led to IOR when onset targets were used. This pattern of results is attributed to feature-dependent top-down contingent capture, meaning that attention is only shifted toward a cue if the cue happens to match a top-down set of searched-for relevant target features (Folk et al., 1992). Past research has shown similar principles for color search. If participants search for a specific target color (such as for blue), attention is also often only shifted towards cues of this searched-for color (i.e., blue cues) but not (or less so) to singleton cues of an irrelevant color (e.g., green cues; Ansorge, Kiss, Worschech, \& Eimer, 2011; Eimer \& Kiss, 2008; Folk \& Remington, 1998; Gibson \& Amelio, 2000; for a review see Burnham, 2007). When tested by Gibson and Amelio, color singletons did never lead to IOR even if these color-singletons were of a top-down matching color - that is, when the cues were of the same color as the searched-for target colors. However, the mere match to the color-defined task set may not have been sufficient for the otherwise irrelevant color cues to produce IOR.

Therefore, in the current experiments we went one step further. Rather than using cues that were uncorrelated to the position of the discrimination targets, our participants were forced to attend to the color-singleton cues in at least half of the trials of Experiment 1 to find a discrimination target at this position. This is an even stronger relevance manipulation than the one used by Gibson and Amelio who used top-down matching cues that could otherwise be ignored. In this way, we wanted to create maximally sensitive conditions to reveal whether color singletons elicited saccadic IOR. To anticipate the results of our first experiment, we found saccadic IOR after the color singletons. We therefore also ran a control experiment to test whether attention and cue relevance was indeed responsible for saccadic IOR - that is, whether saccadic IOR could be successfully prevented if no attention shift to the first cue was required.

\section{Experiment 1}

On each trial, three successive displays were shown and participants had two different tasks, a perceptual discrimination task and a saccade task (see Figure 1). The first display (cue display) contained a blue colorsingleton cue presented among five gray distractors. The participants had to (covertly) select the cued position for subsequent discrimination of a target shape at this position. The discrimination target was shown $500 \mathrm{~ms}$ after the blue singleton cue. Together with the discrimination target, a red color-singleton was shown that served as a cue for the saccade that had to be made upon appearance of the third display (saccade display) another $500 \mathrm{~ms}$ later. Because the position of the red saccade cue was uncorrelated to the position of the blue cue and the stimulus-onset asynchrony (SOA) between the blue cue and the saccade display was sufficiently long (1 s), we expected to find saccadic IOR in saccadic reaction times (RTs) and with respect to the position of the blue color-singleton cue in the first display.

We also included $50 \%$ control trials, in which a green singleton cue was presented instead of the blue singleton cue. The green singleton cue signaled to the participants that no shape discrimination was required in the subsequent display and therefore the position of the green singleton cue was always irrelevant. It will be interesting to see whether saccadic IOR is weaker under these irrelevant cueing conditions as might be assumed if saccadic IOR is a consequence of an attention shift and if attention is only shifted to the searched-for relevant cue.

\section{Methods}

Participants. Ten volunteers (5 female) participated in Experiment 1. Their mean age was 31 years. Here and in Experiment 2, all observers reported normal or corrected-to-normal vision. Written and informed consent was obtained from each participant before the experiment.

Apparatus. Visual stimuli were presented on a 19inch CRT color monitor (Sony Multiscan G400), with a screen resolution of $1,024 \times 768$ pixels. Its refresh rate was $100 \mathrm{~Hz}$. The participants sat at a distance of $57 \mathrm{~cm}$ from the screen in a quiet, dimly lit room, with their head resting on a chin rest to ensure a constant viewing distance and a straight-ahead gaze direction. Manual responses for the discrimination task were registered via a 
standard keyboard, placed directly in front of the observers. Participants' manual responses were collected by the keys \#F and \#J (labeled "left" and "right"). Saccades were recorded with an EyeLink 1000 Desktop Mount system (SR Research, Mississauga, Ontario, Canada) with a $35 \mathrm{~mm}$ lens, and EyeLink Software version 4.52, sampling at maximal resolution. Eyetracking was monocular from the dominant eye. A 9-point calibration was used to adjust the eyetracker before the experiment and in advance of every single block.

Stimuli and Procedure. Three successive displays were shown on each trial (see Figure 1). A central fixation cross was visible throughout each trial. All objects on the screen were equi-luminant $\left(\sim 30 \mathrm{~cd} / \mathrm{m}^{2}\right)$. The first display (cue display) was presented for $50 \mathrm{~ms}$. It consisted of six equidistant digital letter- 8 placeholders (with size of $1.7^{\circ} \times 1.0^{\circ}$, stroke strength of $0.3^{\circ}$ ), presented on the circumference of a virtual circle with an eccentricity of $7.0^{\circ}$ centered on the screen center. The figures were located at 2, 4, 6, 8, 10 and 12 o'clock positions. Five digits were presented in gray (CIELAB color coordinates: $6.9,16.8$ ) and one was presented in a different color (color singleton cue), either in blue $(46.9,-89.0)$ or in green $(-30.2,24.9)$. The color singleton cue was always shown at one of the four lateral positions. It was never presented on the vertical meridian - that is, it was never above or below fixation (6, and 12 o'clock). The color of the singleton indicated the task: A blue singleton signaled that the discrimination task had to be performed while the green singleton could be ignored.

After an inter-stimulus interval of $450 \mathrm{~ms}$ (blank screen), the discrimination display was presented for 50 ms. At the positions of the figure- 8 placeholders three letters "E" and three digits " 3 " were now presented in digital notation. Five of these shapes were presented in gray, one was presented in red $(47.6,41.1)$. Similar as for the color singleton in the cue display, the red singleton cue could likewise only appear at one of the four lateral positions (never above or below fixation). In this display, one figure served as a discrimination target if it had been cued by a blue singleton in the preceding display. The red singleton indicated where the saccade should be directed upon appearance of the subsequent display. Positions of the first cue (blue or green) and the second (red) cue were uncorrelated across trials. As both cues were only presented at the four lateral positions, there were $25 \%$ trials with first and second cue at the same position (SP condi- tion) and $75 \%$ trials with first and second cue at different positions (DP condition). Participants were explicitly informed about these contingencies.

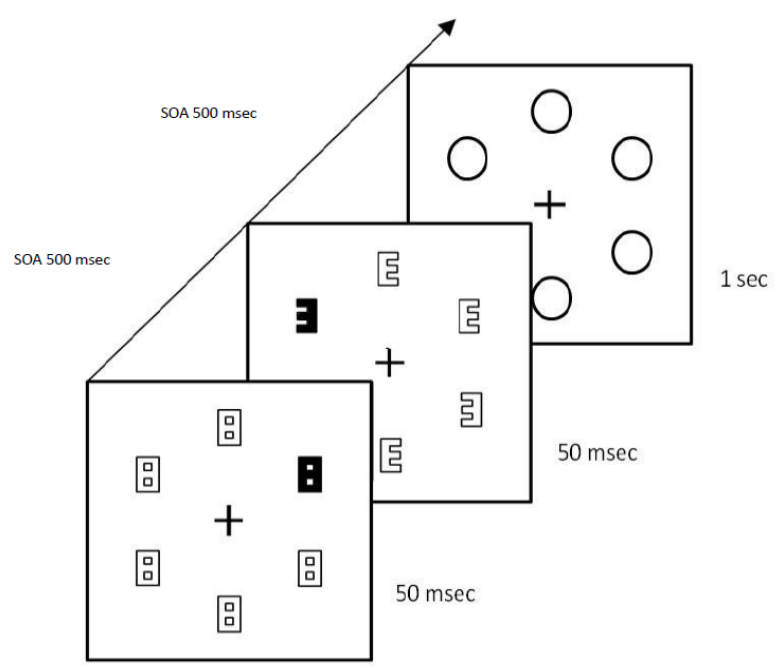

Figure 1: Depicted is an example of a different-position (DP) trial. The first (lower) display was the cue display, in which a color singleton cue (illustrated as a black figure-8) was presented; a blue singleton indicated the position of the subsequent discrimination target (relevant cue); a green singleton indicated that no discrimination task followed (irrelevant cue). The second (middle) display was the discrimination display; following a blue singleton cue, participants had to memorize the identity (figure-E or figure 3) at the position previously occupied by the blue cue. Alongside the discrimination target, we presented a red color singleton as saccade cue (depicted as a black figure). The third (upper) display was the saccade display; participants had to saccade to the target ring at the position previously indicated by the red saccade cue. The arrow illustrates the temporal sequence. Stimuli are not drawn to scale. SOA = Stimulus Onset Asynchrony.

After another blank inter-stimulus interval of $450 \mathrm{~ms}$ the saccade display was presented, containing six empty circles surrounding the same stimulus positions as were used in the preceding displays. The onset of this screen was the go signal for the saccade. The saccade display was presented for $1 \mathrm{~s}$.

After the saccade was executed, participants pressed the key corresponding to the identity of the discrimination target letter on a standard keyboard. If no discrimination was necessary (green singleton), this part 
of the trial was skipped. Participants started the next trial in a self-pace manner by pressing the space bar. $500 \mathrm{~ms}$ elapsed before presentation of the next cue display.

Blocks consisted of 64 trials and feedback was given about whether the target discrimination was correct and about whether the saccade was registered during the third screen. Altogether ten blocks were run, the first block was considered as training. Within a block of trials, the different combinations of the discrimination target ( $E$ or $3)$, first cue position $(2,4,8$, or 10 o'clock), first cue color (blue, green), and second cue's position $(2,4,8$, or 10 o'clock) were equally likely and presented in a pseudorandom order.

For the analysis of the performance, data were collapsed across different target types (E vs. 3) and different target positions $(2,4,8$, and 10 o'clock). In the analyses, the variables of interest were whether the cue was relevant (blue) or irrelevant (green) and whether the target was shown at the same position (SP) as the cue or at a different position (DP) than the cue. (For the latter DP condition, data were collapsed across the different conceivable cue-target position combinations.)

\section{Results}

Saccadic RT was calculated as the time between the onset of the saccade target and the time of the local velocity minimum immediately preceding the point at which eye velocity exceeded $80 \%$ s. Trials with eye movements during the cueing displays and with saccades executed faster than $100 \mathrm{~ms}$ after the onset of the saccade screen or later than $1 \mathrm{~s}$ after onset of the saccade screen were rejected (4.9\% of all trials). Of the remaining SP trials, $12.0 \%$ were discarded because they did not land on the target (i.e., outside an area of $1.5^{\circ}$ around the center of the saccade target), and of the remaining DP trials, $6.6 \%$ had to be discarded for the same reason. This may already speak for an IOR-similar effect but a repeatedmeasures analysis of variance (ANOVA) on the arc-sine transformed rates of error saccades that landed in an area of $1.5^{\circ}$ around the center of the cued distractor, with the two variables position (SP vs. DP) and first cue type (relevant or irrelevant), only revealed a tendency towards a main effect of position, $F(1,9)=3.84, p=.08$. The main effect of first cue type, $F(1,9)=3.32, p=.10$, and the interaction, $F(1,9)=2.99, p=.12$, were also not significant. See Table 1 for the results.
Next, we used the mean correct saccadic RTs (corrected for outliers exceeding 2 SDs from the mean, per individual and condition) for an ANOVA with the variables position (SP vs. DP) and first cue type (relevant or irrelevant). This ANOVA led to a significant position effect, $F(1,9)=6.64, p<.05$, reflecting saccadic IOR that is, lower saccadic RTs in DP $(\mathrm{M}=308 \mathrm{~ms})$ than SP $(\mathrm{M}=338 \mathrm{~ms})$ conditions. The variable first cue type, $F(1$, $9)=3.05, p=.12$, and the interaction, $F(1,9)=1.32, p=$ .28 , were both not significant. For the results see also Figure 2.

Table 1

Error rates and arc-sine transformed error rates of saccades as a function of cue position (same as targets or different from target) and of cue type (relevant or irrelevant) in Experiment 1.

\begin{tabular}{ccc}
\hline & ER & ER $(\arcsin )$ \\
\hline SP relevant & 0.092 & 0.093 \\
DP relevant & 0.283 & 0.322 \\
SP irrelevant & 0.076 & 0.076 \\
DP irrelevant & 0.111 & 0.111 \\
\hline
\end{tabular}

Note. SP: same position; DP: different position; ER: Error rate; arcsin: arcus-sinus transformed.

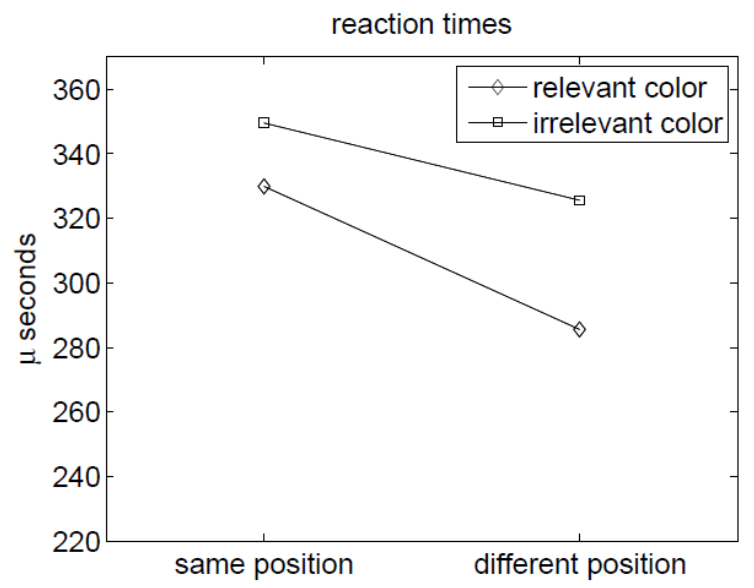

Figure 2: Saccadic reaction times (SRTs; in milliseconds) in Experiment 1 as a function of the first cue's color, and the relation between cue and target positions.

Thereafter, we ran a complementary analysis to test whether the conclusions were qualified for different parts of the saccadic RT distribution. For this ANOVA, all correct saccadic RTs were vincentized: Separately for each participant and conditions, saccadic RTs were sorted from fastest to slowest and grouped into five percentiles 
or time-bins. A repeated-measures ANOVA with the variables position (SP vs. DP), first cue type (relevant or irrelevant) and percentile revealed a significant main effect of position, $F(1,9)=5.45, p<.05$. Average saccadic RT for trials with both cues presented at the same position was slower than for trials with both cues on different positions (349 ms vs. $321 \mathrm{~ms}$ ), indicating saccadic IOR. With the exception of a trivial main effect of percentile, $F(1,9)=40.17, p<.01 \quad$ (saccadic RTs increasing with percentile), no other significant effects or interactions were found, all $F \mathrm{~s}<1.80$, all $p \mathrm{~s}>.20$. See also Figures 3 and 4 for the results.

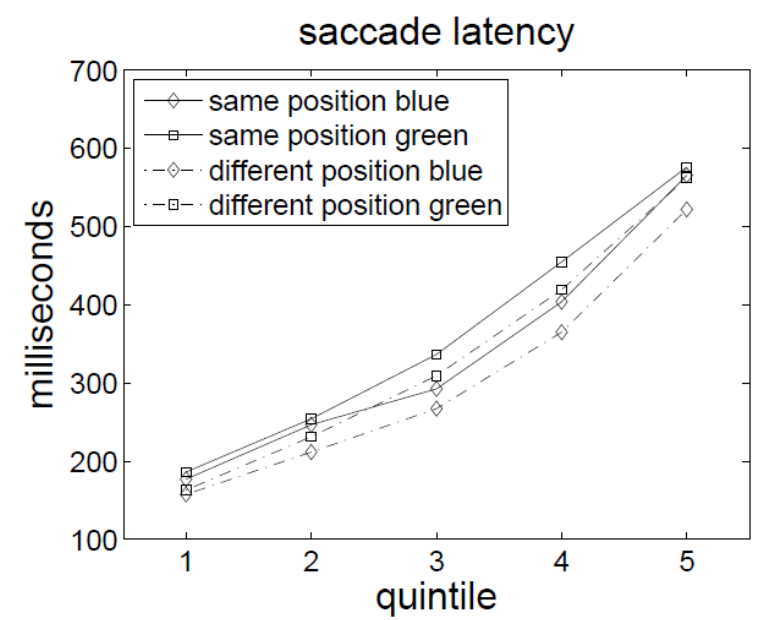

Figure 3: Saccadic reaction times (SRTs; in milliseconds) in Experiment 1 as a function of the first cue's color, the relation between cue and target positions, and the SRT quintile.

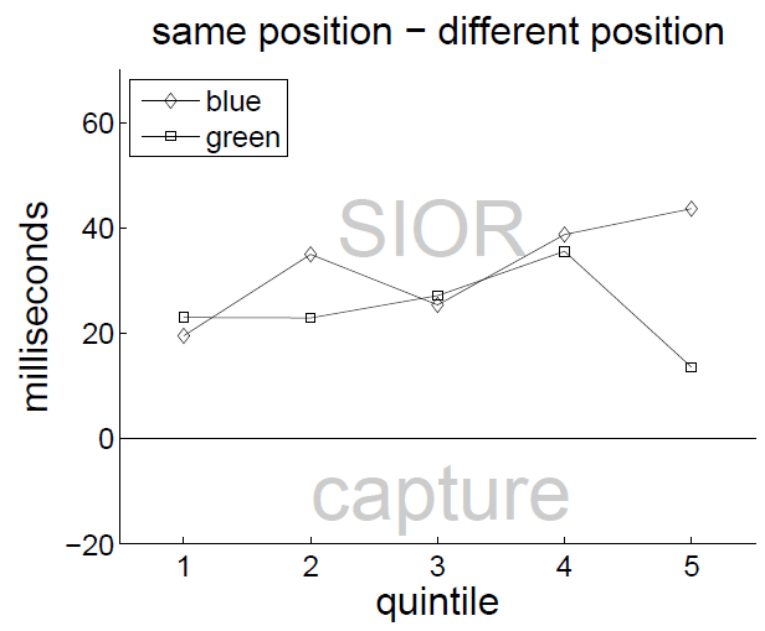

Figure 4: SRT difference between same position trials and different position trials as a function of the first cue's color, and the SRT quintile in Experiment 1; positive values denote saccadic inhibition of return (SIOR); negative values denote attention capture.

We also tested whether attention capture was reflected in the discrimination performance in the relevant conditions - that is, whether the cue-target position relation affected the discrimination performance. This was the case. Performance was better in SP (correct discrimination rate $=84 \%$ ) than DP (correct discrimination rate $=$ $78 \%$ ) conditions, $F(1,9)=10.87, p<.01$.

\section{Discussion}

Experiment 1 showed clear saccadic IOR after relevant color singleton cues. This is different from prior studies with manual responses that have failed to find IOR after uninformative color singletons (Gibson \& Amelio, 2000; Pratt et al., 2001). However, our results are consistent with findings by Godijn and Theeuwes (2004) who found a small saccadic IOR effect after a relevant color singleton.

Also of interest, the present saccadic IOR effect was not affected by whether or not the position of the singleton cue in the first display had been relevant (blue, i.e., signaling the position of the discrimination target) or irrelevant (green) for the discrimination task in a given trial. In a way, this result is not very surprising, given that participants had to attend to both cues in order to determine whether the discrimination task had to be done. Thus, both cue colors could be regarded task-relevant. The fact that for one cue, not only its color, but its position was additionally crucial for the discrimination task did not change the magnitude of saccadic IOR. Our finding is in accordance with either stimulus-driven attention capture by all singleton cues (Theeuwes, 2010) or it could reflect singleton capture according to a top-down singleton search set (Bacon \& Egeth, 1994). In any case, the results do not support the top-down feature search explanation (Folk et al., 1992). If the participants had searched for only relevant blue cues, saccadic IOR being conditional on a preceding attention shift should only have occurred with the blue but not with the green cues.

Discrimination performance likewise showed an effect of cue position. Discrimination performance was better in SP than DP conditions. Remember that the discrimination target was presented $500 \mathrm{~ms}$ before the imperative signal for saccade execution. Thus, the finding is 
likely to reflect the usually found initial attentional capture effect that later turns into inhibition.

Jointly, our results suggest that it is possible to find at least saccadic IOR after relevant color singletons. Experiment 2 therefore examines whether cue relevance is crucial to obtain saccadic IOR. Further, Experiment 2 allows us to exclude a caveat to our argument that results are driven by attentional mechanisms. One could argue that the saccadic IOR effect in Experiment 1 was not due to an attention shift to the first cue. Instead, the first color-singleton cue (blue or green) might have masked the (detection of the color of) the subsequent red cue in the SP conditions. In contrast, less or no masking would have occurred with first and second cue at different positions. In other words, the first cue may have created some sort of forward masking effect, delaying the detection of the second cue in SP but not in DP conditions. This could have created a saccadic RT cost that would not reflect an attention shift to the first cue. This possibility was tested in Experiment 2.

\section{Experiment 2}

Experiment 2 served as a control experiment to examine the effects of uninformative cues and to exclude that results of Experiment 1 reflect forward masking effects. Participants had to attend only to the red cue in the discrimination display, and could ignore the blue or green cue in the first display. This was achieved by asking the participants to only search for the red cue, discriminate the target at its position and to subsequently saccade to that same position with onset of the saccade display.

Under these conditions, a saccadic IOR effect that is conditional on a preceding attention shift to the first blue or green cue should be prevented. With only one relevant color (here: the color red), participants should be able to search for this red color in a top-down manner with a feature template (Folk \& Anderson, 2010). As a consequence, the irrelevant blue and green cue should no longer capture attention.

Our predictions are straightforward. If participants adopt a feature search template and only search for red cues, we expect no capture of attention by the nonmatching blue and green cues and, hence, no subsequent saccadic IOR conditional on such preceding attention shifts. In contrast, if location-specific masking of the sec- ond cue in SP conditions created a sensory delay of the detection of the red cue in Experiment 1, we would predict likewise longer saccadic RTs in SP conditions than DP conditions in Experiment 2 - that is, saccadic IOR.

Two further accounts predict saccadic IOR for Experiment 2. First, if the blue and green color singletons capture attention in a stimulus-driven way, we should find saccadic IOR. Second, theoretically it is also still possible for the participants to search for the target with a singleton-search template, which should likewise produce saccadic IOR.

To summarize the predictions: We can reject an explanation of Experiment 1's saccadic IOR effect in terms of masking by the color switch if the saccadic IOR effect was abolished in the present experiment. In turn, the conclusion that capture in the first display was critical for saccadic IOR would be supported. If, however, saccadic IOR persists in the present experiment, we cannot reject a color switch explanation of the saccadic IOR effect, but an attentional explanation (stimulus-driven capture or top-down singleton search) of the saccadic IOR effect might also be viable.

\section{Methods}

Participants. Ten volunteers (3 female) with a mean age of 31 years participated in Experiment 2.

Apparatus, Stimuli, and Procedure were the same as in Experiment 1 with the exception that the task changed. Participants had to discriminate the target at and subsequently saccade (in the saccade display) to the position that was indicated by the red cue. Thus, the participants were allowed if not encouraged to completely ignore all blue and green cues that were shown in the cue display, and no re-shifting of attention from these blue or green cues and to the red cues would have been necessary. Note that therefore one variable of interest, cue type, with the steps relevant and irrelevant, was irrelevant in all of the conditions. However, for the sake of consistency of the analyses the variable cue type was again conceptualized as a two-step variable, with the steps blue cue (corresponding to the relevant cue in Experiment 1) and green cue (corresponding to the irrelevant cue in Experiment 1).

\section{Results}

As in Experiment 1, saccades faster than $100 \mathrm{~ms}$ or slower than $1 \mathrm{~s}$ were discarded (10.1\%). Of the remaining SP saccades, $7.8 \%$ were not directed to the saccade tar- 
get, while of the remaining DP saccades $6.8 \%$ landed at the wrong position. A repeated-measures ANOVA of the erroneous saccades to the cued distractor, with the variables position (SP vs. DP), and cue type (here: color of the first cue: blue or green), did not reveal any significant main effects or interactions, all $F \mathrm{~s}<1.00$. For the results see Table 2 .

\section{Table 2}

Error rates and arc-sine transformed error rates of saccades as a function of cue position (same as targets or different from target) and of cue type (relevant or irrelevant) in Experiment 2.

\begin{tabular}{ccc}
\hline & ER & ER $(\arcsin )$ \\
\hline SP relevant & 0.156 & 0.159 \\
DP relevant & 0.176 & 0.179 \\
SP irrelevant & 0.157 & 0.161 \\
DP irrelevant & 0.190 & 0.195 \\
\hline
\end{tabular}

Note. SP: same position; DP: different position; ER: Error rate; arcsin: arcus-sinus transformed.

Next, we used the mean correct saccadic RTs (corrected for outliers exceeding 2 SDs from the mean, per individual and condition) for an ANOVA with the variables position (SP vs. DP) and first cue type (blue vs. green). This ANOVA led to no significant main effects, both $F \mathrm{~S}<1.00$, and to no significant interaction, $F(1,9)=$ $1.78, p=.22$. For the results see also Figure 5 .

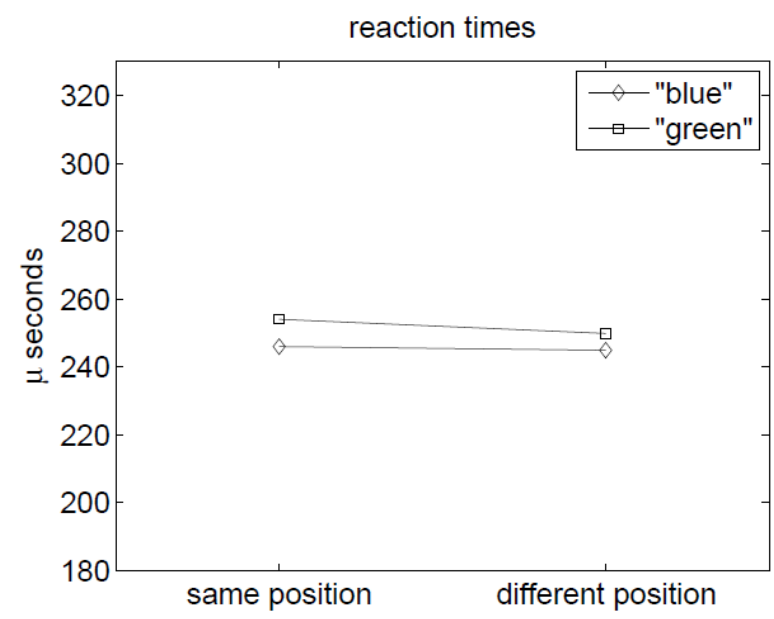

Figure 5: Saccadic reaction times (SRTs; in milliseconds) in Experiment 2 as a function of the first cue's color, and the relation between cue and target positions.

Again, a repeated-measures ANOVA of the correct saccadic RTs, with the variables position (SP vs. DP), first cue type (blue vs. green), and percentile (or timebin) confirmed the conclusion. It indicated no significant effect for cue position: Although saccadic RTs were slower when both cues were presented at the same position than at different positions ( $278 \mathrm{~ms}$ vs. $271 \mathrm{~ms})$, this effect failed to reach significance, $F(1,9)=3.06, p=.11$. Besides percentile, no other main effect or two-way interaction was significant either. Only the three-way interaction of cue position, first cue type, and percentile was significant, $F(4,36)=2.88, p<.05$. This interaction was due to faster saccadic RTs for different-position greencue and same-position blue-cue conditions compared to same-position green-cue and different-position blue-cue conditions in the fifth percentile. In other words, the green cue produced an effect reminiscent of saccadic IOR in the fifth percentile, whereas the blue cue produced an inversed saccadic RT pattern. See also Figures 6 and 7 for the results.

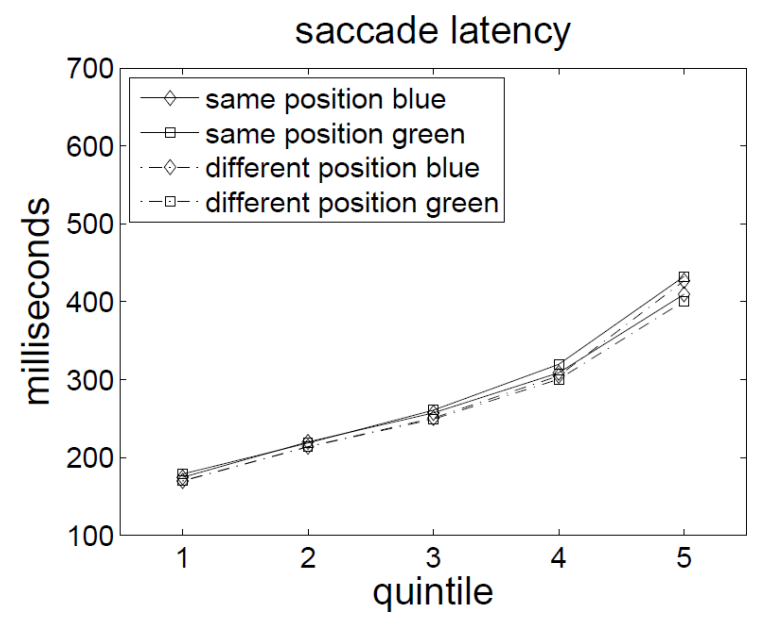

Figure 6: Saccadic reaction times (SRTs; in milliseconds) in Experiment 2 as a function of the first cue's color, the relation between cue and target positions, and the SRT quintile.

As there was a trend towards saccadic IOR in the present experiment, too, we also ran one additional $t$-test to compare SP's with DP's saccadic RTs, with the data collapsed across the two irrelevant conditions of the present experiment and with the data from the irrelevant condition of Experiment 1 (to increase the number of observations, $n$, and thus, the statistical power of the test). However, there was no significant difference between the SP and DP conditions, $t(19)=1.45, p=.16$.

Again, we tested whether attention capture was reflected in better SP than DP conditions. This was not the 
case. Across all conditions, performance was not significantly better in SP (correct discrimination rate $=90 \%$ ) than DP (correct discrimination rate $=89 \%$ ) conditions, $F(1,9)=3.56, p=.09$.

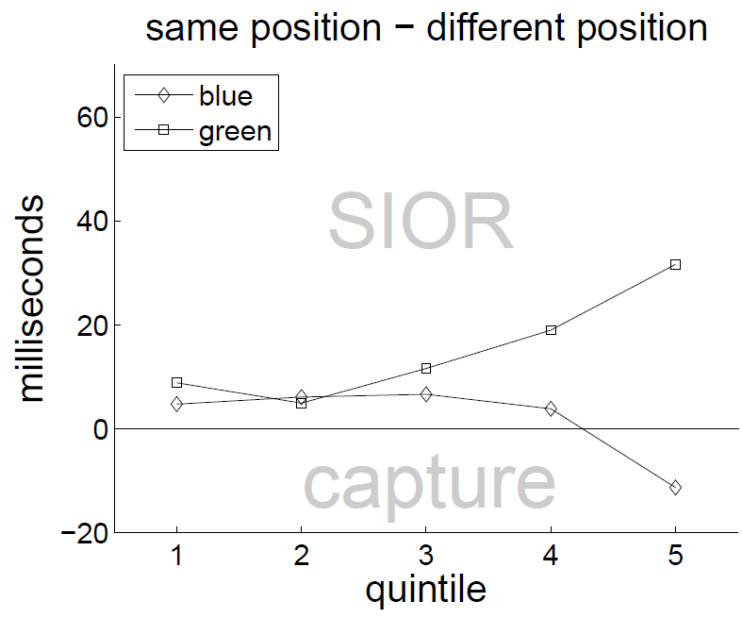

Figure 7: SRT difference between same position trials and different position trials as a function of the first cue's color, and the SRT quintile in Experiment 2; positive values denote saccadic inhibition of return (SIOR); negative values denote attention capture.

\section{Discussion}

Changing the task instructions led to the elimination of saccadic IOR in (almost) all conditions. This result falsifies the notion that multiple color changes at the same position (i.e., masking) could be responsible for worse cue detection. In turn, the absence of the saccadic IOR effect is in line with an attentional explanation of saccadic IOR in Experiment 1. We argued that the capture of attention to the blue and green cues in Experiment 1 was responsible for SIOR in the saccade display. By asking our participants to only search for the red cue in Experiment 2, we apparently prevented attention shifts to the green and blue cues and thus, also prevented saccadic IOR. Accordingly, discrimination performance did likewise not differ between SP and DP trials.

We did find a residual saccadic IOR effect in the slowest saccadic RTs after the green cues. This residual cost might indeed reflect a contribution of sensory interference due to the color change from green to red, however, it seems, only on a small subset of trials. Importantly, for most trials, no saccadic IOR effect was found.

\section{General Discussion}

In the current study, we found saccadic IOR after relevant color singletons (Experiment 1). This finding is in contrast to previous studies that have failed to find IOR with irrelevant color singletons after manual responses (Gibson \& Amelio, 2000; Pratt et al., 2001). Thus, one decisive prerequisite to obtain (saccadic) IOR with color singletons may be that the singleton cue provides taskrelevant information. In the present Experiment 1, the color of the cue indicated whether the discrimination task had to be performed and, in case of a blue cue, at which position. In contrast, the cue in Experiment 2, similarly to previous studies on IOR after color singletons (Gibson \& Amelio, 2000; Pratt et al., 2001), was completely irrelevant for the subsequent discrimination and saccade tasks. In line with previous studies, IOR was abolished.

Another important difference between the present study and previous studies concerned the use of saccades in the current investigation as compared to manual responses in previous studies lacking an IOR effect. The use of saccades is very likely a decisive difference assuming that IOR seems to reflect selection for action, at least to some extent (Klein, 2000; Klein \& Pontefract, 1994). Accordingly, IOR tends to be larger with overt shifts (or saccades) than with covert shifts of attention (Pratt \& Neggers, 2008). Also, in line with this explanation, the best evidence for (small) IOR effects after color singletons using RTs as dependent measure stems from a study that used saccades (Godijn \& Theeuwes, 2004). With this in mind, it remains somewhat open whether cue relevance is as crucial for IOR to emerge in manual RTs as it is for saccadic RTs. More research will be required to resolve the contradictory results found for manual RTs (Gibson \& Amelio, 2000; Pratt et al., 2001; Theeuwes \& Chen, 2005; Theeuwes \& Godijn, 2002).

IOR (and saccadic IOR) has sometimes been attributed to other factors than (overt) attention. Some authors argue that IOR reflects sensory adaptation (Dukewich, 2009; Hu, Samuel, \& Chan, 2011). According to this account, inhibition is stronger whenever features are repeated. This inhibition can be observed for spatial position repetitions, as with the standard IOR effect, as well as for non-spatial feature repetitions, such as the repetition of a particular color (e.g., Hu et al., 2011). For two reasons, sensory adaptation effects did probably not play a major role in the present study. First, we observed saccadic IOR after color changes rather than after color repe- 
titions. In Experiment 1, either a blue or a green cue was presented in the first display, whereas a red cue was shown in the second display. Also, the saccade target was yet of another color (gray). These color-change conditions would not be favorable for a feature-adaptation effect. Second, when we changed the task and asked our participants to only search for the red cues, saccadic IOR was eliminated (Experiment 2). It would be difficult to understand how this change in task instruction should have abolished a task-independent automatic adaptation effect.

Other authors have emphasized that IOR could reflect motor activation (Hunt \& Kingstone, 2003; Lupiàñez, 2010; Taylor \& Klein, 2000). In line with this assumption, for example, saccadic IOR is affected by variables that do not impact on covert attention effects (Hunt \& Kingstone, 2003; but see Souto \& Kerzel, 2009). This explanation of IOR in terms of motor activation is, however, not at variance with our view. In fact, we believe that much of the saccadic IOR effects reflected selection for action. We propose that saccadic IOR after color singletons may reflect the inhibition of overt attention (i.e., inhibition of executing an eye movement to prevent oculomotor capture) following a covert attention shift.

Finally, we want to address two remaining questions. First, which principles may account for the different results in Experiment 1 and 2? Second, how is saccadic IOR by color singletons brought about if the SC is colorinsensitive? Concerning the first question, we have argued that saccadic IOR after relevant blue and irrelevant green cues in Experiment 1 could have reflected either stimulus-driven attention capture by any salient stimulus (Theeuwes, 1992) or attention shifts contingent on a topdown search for a singleton (Bacon \& Egeth, 1994). Results of Experiment 2 seem to contradict the stimulusdriven account. In Experiment 2, our participants were asked to search for the red cue. This was evidently enough to overcome capture and saccadic IOR by both color singleton cues in the first display. Accordingly, it seems feasible that our participants were able to choose between one of two top-down search modes: top-down search with a feature template (as in Experiment 2) or top-down search for singletons (as in Experiment 1). However, it is still possible that the participants in Experiment 1 simply abandoned their top-down search settings: With two relevant colors, participants might have found it too difficult to search for two features in parallel and might simply not have used any kind of top-down control over their search. Thus, we cannot exclude that saccadic IOR in Experiment 1 reflects stimulus-driven attention capture, whereas the lack of saccadic IOR in Experiment 2 reflects top-down influences.

Concerning the second question, it is likely that the $\mathrm{SC}$ is not the only structure involved in IOR, but there may also be cortical areas, for example, the posterior parietal cortex (PPC) (Tian, Klein, Satel, Xu, \& Yao, 2011). The PPC is assumed to also contribute for instance to color salience effects (Arcizet, Mirpour, \& Bisley, 2011). In addition, recent evidence suggests that some SC neurons are actually highly sensitive to information originating from chromatic pathways, albeit color information arrives with a certain delay compared to achromatic signals (White, Boehnke, Marino, Itti, \& Munoz, 2009). Interestingly, Fecteau \& Munoz (2005) report two distinct IOR-related modulations in SC neuron activity, one early, one late. They conclude that different components of IOR originate at early and late processing stages. We speculate that IOR after color singletons may originate at late processing stages. In sum, IOR seems to be mediated by more than one pathway or brain area, allowing for IOR effects to occur even after color singletons.

\section{Acknowledgements}

Supported by project number CS11-009 of the WWTF (Wiener Wissenschafts- und Technologiefonds) to Ulrich Ansorge, Otmar Scherzer, and Shelley Buchinger and by project number 100014_140379 of the Swiss National Science Foundation to Sabine Born and Dirk Kerzel. We thank Sabine Schirk for help with the data collection. Correspondence concerning this article should be addressed to Heinz-Werner Priess, Faculty of Psychology, University of Vienna, Liebiggasse 5, 1010 Vienna, Austria.

E-mail: heinz-werner.priess@univie.ac.at

\section{References}

Adamo, M., Pun, C., \& Ferber, S. (2010). Multiple attentional control settings influence late attentional selection but do not provide an early attentional filter. Cognitive Neuroscience, 1, 102-110. 
Allport, A. (1987). Selection for action: some behavioral and neurophysiological considerations of attention and action. In H. Heuer \& A. F. Sanders (Eds.), Perspectives on perception and action (pp. 395-419). Hillsdale, NJ: Erlbaum.

Ansorge, U., \& Heumann, M. (2004). Peripheral cuing by abrupt-onset cues: the influence of color in S-R corresponding conditions. Acta Psychologica, 116(2), 115143.

Ansorge, U., \& Heumann, M. (2004). Peripheral cuing by abrupt-onset cues: the influence of color in S-R corresponding conditions. Acta Psychologica, 116(2), 115143.

Ansorge, U., Kiss, M., Worschech, F., \& Eimer, M. (2011). The initial stage of visual selection is controlled by top-down task set: new ERP evidence. Attention, Perception \& Psychophysics, 73, 113-122.

Arcizet, F., Mirpour, K., \& Bisley, J. W. (2011). A pure salience response in posterior parietal cortex. Cerebral Cortex, 21, 2498-2506.

Bacon, W. F., \& Egeth, H. E. (1994). Overriding stimulus-driven attentional capture. Perception \& Psychophysics, 55(5), 485-496.

Burnham, B. R. (2007). Displaywide visual features associated with a search display's appearance can mediate attentional capture. Psychonomic Bulletin \& Review, 14, 392-422.

Deubel, H., \& Schneider, W.X. (1996). Saccade target selection and object recognition: evidence for a common attentional mechanism. Vision Research, 36, 1827-1837.

Dukewich, K. R. (2009). Reconceptualizing inhibition of return as habituation of the orienting response. Psychonomic Bulletin \& Review, 16, 238-251.

Duncan, J., \& Humphreys, G. W. (1989). Visual search and stimulus similarity. Psychological Review, 96, 1481-1490.

Eimer, M., \& Kiss, M. (2008). Involuntary attentional capture is determined by task set: Evidence from event-related brain potentials. Journal of Cognitive Neuroscience, 20, 1423-1433.

Fecteau, J. H., \& Munoz, D. P. (2005). Correlates of capture of attention and inhibition of return across stages of visual processing. Journal of Cognitive Neuroscience, 17(11), 1714-1727.
Folk, C. L., \& Anderson, B. A. (2010). Targetuncertainty effects in attentional capture: Colorsingleton set or multiple attentional control settings? Psychonomic Bulletin \& Review, 17, 421-426.

Folk, C. L., Remington, R. W., \& Johnston, J. C. (1992). Involuntary covert orienting is contingent on attentional control settings. Journal of Experimental Psychology: Human Perception and Performance, 18, 1030-1044.

Fuchs, I., \& Ansorge, U. (2012). Unconscious cueing via the superior colliculi: Evidence from search for onset and color targets. Brain Sciences, 2, 33-60.

Gibson, B. S., \& Amelio, J. (2000). Inhibition of return and attentional control settings. Perception \& Psychophysics, 62, 496-504.

Godijn, R., \& Theeuwes, J. (2004). The relationship between inhibition of return and saccade trajectory deviations. Journal of Experimental Psychology: Human Perception and Performance, 30, 538-554.

Hickey, C., McDonald, J. J., \& Theeuwes, J. (2006), Electrophysiological evidence of the capture of visual attention. Journal of Cognitive Neuroscience, 18, 604613.

Hu, F. K., Samuel, A. G., \& Chan, A. S. (2011). Eliminating inhibition of return by changing salient nonspatial attributes in a complex environment. Journal of Experimental Psychology: General, 140, 35-50.

Hunt, A. R., \& Kingstone, A. (2003) Inhibition of return: dissociating attentional and oculomotor components. Journal of Experimental Psychology: Human Perception and Performance, 29, 1068-1074.

Itti, L., \& Koch, C. (2000). A saliency-based search mechanism for overt and covert shifts of visual attention. Vision Research, 40(10-12), 1489-1506.

Itti, L., Koch, C., \& Niebur, E. (1998). A model of saliency-based visual attention for rapid scene analysis. IEEE Transactions on Pattern Analysis and Machine Intelligence, 20(11), 1254-1259.

Klein, R. M. (2000). Inhibition of Return. Trends in Cognitive Sciences, 4, 138-147.

Klein, M., \& Pontefract, A. (1994). Does oculomotor readiness mediate cognitive control of visual-attention - revisited. In C. Umiltà \& M. Moscovitch (Eds.), Attention and Performance $X V$ : Conscious and nonconscious information processing (pp. 333-350). Cam- 
bridge, MA: MIT Press.

Lupiàñez, J. (2010). Inhibition of return. In A. C. Nobre \& J. T. Coull (Eds.), Attention and time (pp. 17-34). Oxford: Oxford University Press.

Posner, M. I. (1980). Orienting of attention. Quarterly Journal of Experimental Psychology, 32, 3-25.

Posner, M. I., \& Cohen, Y. (1984). Components of visual orienting. In H. Bouma \& D. G. Bouwhuis (Eds.). Attention and Performance Vol. X, (pp 531-556). Hillsdale, NJ: Erlbaum.

Pratt, J., \& Neggers, B. (2008). Inhibition of return in single and dual tasks: Examining saccadic, keypress, and pointing responses. Perception \& Psychophysics, $70,257-265$.

Pratt, J., Sekuler, A., \& McAuliffe, J. (2001) The role of attentional set on attentional cueing and inhibition of return. Visual Cognition, 8, 33-46.

Rafal, R. D., Calabresi, P. A., Brennan, C. W., \& Sciolto, T. K. (1989). Saccade preparation inhibits reorienting to recently attended locations. Journal of Experimental Psychology: Human Perception and Performance, 15, 673-685.

Remington, R. W., Folk, C. W., \& McLean, J. P. (2001). Contingent attentional capture or delayed allocation of attention? Perception \& Psychophysics, 63, 298307.

Scharlau, I. (2002). Leading, but not trailing, primes influence temporal order perception: Further evidence for an attentional account of perceptual latency priming. Perception \& Psychophysics, 64, 1346-1360.

Souto, D., \& Kerzel, D. (2009). Evidence for an attentional component in saccadic inhibition of return. Experimental Brain Research, 195, 531-540.

Taylor, T. L., \& Klein, R. M. (1998). On the cause and effects of inhibition of return. Psychonomic Bulletin and Review, 5, 625-643.

Taylor, T. L., \& Klein, R. M. (2000). Visual and motor effects in inhibition of return. Journal of Experimental Psychology: Human Perception and Performance, 26, 1639-1656.

Theeuwes, J. (1992). Perceptual selectivity for color and form. Perception \& Psychophysics, 51, 599-606.

Theeuwes, J. (2010). Top-down and bottom-up control of visual selection. Acta Psychologica, 123, 77-99.
Theeuwes, J., \& Chen, C. Y. D. (2005). Attentional capture and inhibition (of return): The effect on perceptual sensitivity. Perception, \& Psychophysics, 67, 1305-1312.

Theeuwes, J., \& Godijn, R. (2002). Irrelevant singleton capture attention: Evidence from inhibition of return. Perception, \& Psychophysics, 64, 764-770.

Theeuwes, J., \& Godijn, R. (2004). Inhibition of return and oculomotor interference. Vision Research, 44, 1485-1492.

Tian, Y., Klein, R. M., Satel, J., Xu, P., \& Yao, D. (2011). Electrophysiological explorations of the cause and effect of inhibition of return in a cue-target paradigm. Brain Topography, 24, 164-182.

van Zoest W., Donk, M., \& Theeuwes, J. (2004). The role of stimulus-driven and goal-driven control in visual selection. Journal of Experimental Psychology: Human Perception and Performance, 30, 746-759.

White, B. J., Boehnke, S. E., Marino, R. A., Itti, L., \& Munoz, D. P. (2009). Color-related signals in the primate superior colliculus. Journal of Neuroscience, 29, 12159-12166.

Wu, S. C., \& Remington, R. W. (2003). Characteristics of covert and overt visual orienting: Evidence from attentional and oculomotor capture. Journal of Experimental Psychology: Human Perception and Performance, 29, 1050-1067.

Wurtz, R. H., \& Albano, J. E. (1980). Visual-motor function of the primate superior colliculus. Annual Review of Neuroscience, 3, 189-226. 